\title{
ST-Elevation Myocardial Infarction With Cardiogenic Shock and Severe Acute Ischemic Mitral Regurgitation Rescued by Primary Coronary Intervention Under Hemodynamic Support With Impella
}

Hiroshi Koiwaya, MD, PhD; Nozomi Watanabe, MD, PhD; Kensaku Nishihira, MD, PhD; Yuhei Goriki, MD; Yoshisato Shibata, MD
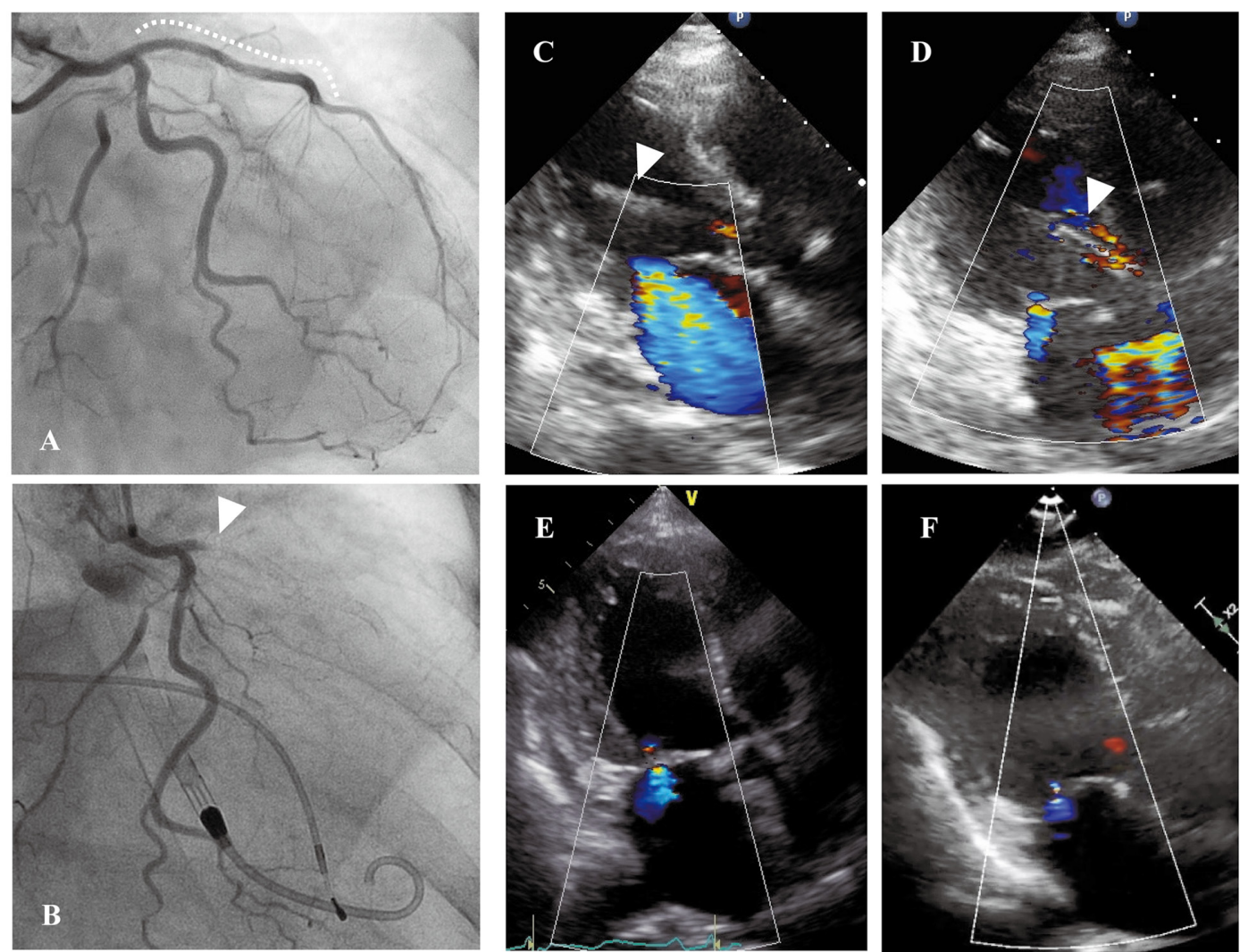

Figure. (A) A 2.5-mm $\times 48-m m$ everolimus-eluting stent (white dotted line) was successfully implanted to the proximal left anterior descending artery (LAD) for stable angina pectoris. (B) Emergency coronary angiography after introduction of the Impella revealed the subacute stent thrombosis at the proximal part of the stent (white arrowhead). (C,D) Severe ischemic mitral regurgitation (IMR; white arrowheads) in the emergency room $(\mathbf{C})$ was significantly reduced immediately after running of the Impella 2.5 (D). (E) Severe hypokinesis in the LAD territory at the onset (left ventricular ejection fraction [LVEF] 20\%) improved 2 weeks after primary percutaneous coronary intervention (PCl; LVEF 52\%), resulting in a significant improvement in IMR. (F) Left ventricular systolic function was preserved and IMR was barely observed at the 1-year follow-up after primary PCI.

Received December 28, 2020; revised manuscript received March 7, 2021; accepted March 23, 2021; J-STAGE Advance Publication released online April 10, 2021 Time for primary review: 57 days

Cardiovascular Center, Miyazaki Medical Association Hospital, Miyazaki, Japan

Mailing address: Hiroshi Koiwaya, MD, PhD, Cardiovascular Center, Miyazaki Medical Association Hospital, 1173 Arita, Miyazaki 880-2102, Japan. E-mail: koiwaya.hiroshi@gmail.com

All rights are reserved to the Japanese Circulation Society. For permissions, please e-mail: cr@j-circ.or.jp

ISSN-2434-0790

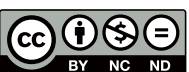


A 62-year-old female returned to the Cardiovascular Center, Miyazaki Medical Association Hospital by air ambulance due to ST-elevation myocardial infarction (STEMI) with severe cardiogenic shock 3 days after percutaneous coronary intervention (PCI). She had successfully undergone drug-eluting stent implantation for a diffuse calcified lesion in the left anterior descending artery (LAD; Figure A). Emergency coronary angiography identified the thrombotic in-stent occlusion under dual antiplatelet therapy with aspirin and clopidogrel (Figure B). Due to the marked hemodynamic deterioration, an Impella (ABIOMED, Danvers, MA, USA) was immediately inserted. Following intracoronary thrombus aspiration and balloon dilatation, coronary flow of Thrombolysis in Myocardial Infarction (TIMI) Grade 3 was achieved.

Serial echocardiography images are shown in Figure $\mathbf{C}-\mathbf{F}$. Severe acute ischemic mitral regurgitation (IMR) with extensive wall motion abnormality in the LAD territory was observed in the acute phase. Immediately after running of the Impella, IMR was barely observed. Left ventricular (LV) systolic function markedly improved 2 weeks after the revascularization under Impella support, and was maintained in the chronic phase.

A recent study reported that LV unloading with the Impella before PCI improved mortality by minimizing the infarct size. ${ }^{1}$ This is the first report showing that LV unloading with the Impella before PCI improved LV systolic function and IMR in a STEMI patient with a stent thrombosis complicated by cardiogenic shock. The procedures in this study were performed in accordance with the Declaration of Helsinki.

\section{Disclosures}

None.

\section{Reference}

1. Kapur NK, Alkhouli MA, DeMartini TJ, George ZH, Goodwin MJ, Josephy N, et al. Unloading the left ventricle before reperfusion in patients with anterior ST-segment-elevation myocardial infarction. Circulation 2019; 139: 337-346.

\section{Supplementary Files}

Please find supplementary file(s);

http://dx.doi.org/10.1253/circrep.CR-20-0131 